\title{
SUSTAINABLE GREEN VENTURE
}

\author{
Sai Mahaan Gannavarapu \\ Graduate BE. (Hons) Mechanical Engineering \\ Birla Institute of Technology \& Science Pilani, Dubai
}

Abstract- Companies and people are hooked to advertisements. Companies are constantly designing and testing out new advertisements to get the public hooked, and they have massive budgets. Our offering to the evergrowing market of Advertisements is to provide a novel solution, by creating a whole new experience at $\mathbf{E}$ charging stations. With more people investing in Electric vehicles as an alternate to traditional fossil fueled cars not because of the government subsidies but due to the far sightedness to create a more conscious planet, we see this potential to use the public EV charging stations to put up self-sufficient off the grid solar AD boards. State of the art technology would showcase adverts to these visionary people, that would benefit them in one way or the other.

Keywords - Electric Vehicle Chargers, Advertisements, Market, AD Boards,

\section{INTRODUCTION}

The last decade saw a major technological trend; some of the greatest excitement and development was focused on the EV industry. EV's have experienced phenomenal growth over the last decade. Global EV sales have exceeded 2 million units by 2018, and by 2020 this figure projected to increase to 4 million. EV's have been offering huge benefits through reduced air pollution with petrol and diesel vehicles.

Most of the companies in the current scenario are keen on advertisements, they are trying out new techniques by designing and experimenting with them to put the audience hooked. The existing problem is that there are a smaller number of advertisement boards which are highly expensive and lack of security cameras at these particular sites. We as a team have come up with a novel solution as the number of electric vehicle users is rising exponentially and we aim to open up new parts of advertisements to run a sustainable green venture. In this day and age, individuals need to see messages conveyed in a medium they know and anticipate that is an expectation for new dynamic information. What superior way to communicate those messages that the digital shows encased with the charging stations that are placed exterior the front of shopping stations or grocery stores for the case. Charging stations are the culminate to publicize to each driver who pulls up to charge. This also provides a unique opportunity for various ad agencies to invest in these advertisements alongside these chargers. These Charging stations can create a 360degree experience allowing customers to look out at the

\section{Addepalli Pranay}

Graduate BE. (Hons) Mechanical Engineering

Birla Institute of Technology \& Science Pilani, Dubai

advertisements. We see this as high potential where the respective EV charging stations can put these solar-powered $\mathrm{AD}$ boards and the people in one way or the other.

\section{DESCRIPTION OF THE MODEL}

The model which is illustrated below comprises how the entire system is operated. The box in green is the Electrical Vehicle Charger. We have 2 RED AD Boards (6ftx $2 \mathrm{ft}$ each) powered by solar panels which are suspended with LED lights so that these could be visible at night. On top of the AD Boards, we have 2 cameras which give us a rough estimate of how many people are using the EV Chargers whether it is weekly, monthly, or yearly. At the back of the EV charger, we have a structure that supports the solar panels. We have the solar panels at the top to give shade to the cars. At the back of the structure, we have a case in which the battery and inverter are placed inside. The case operates on the basis of a lock and key system.

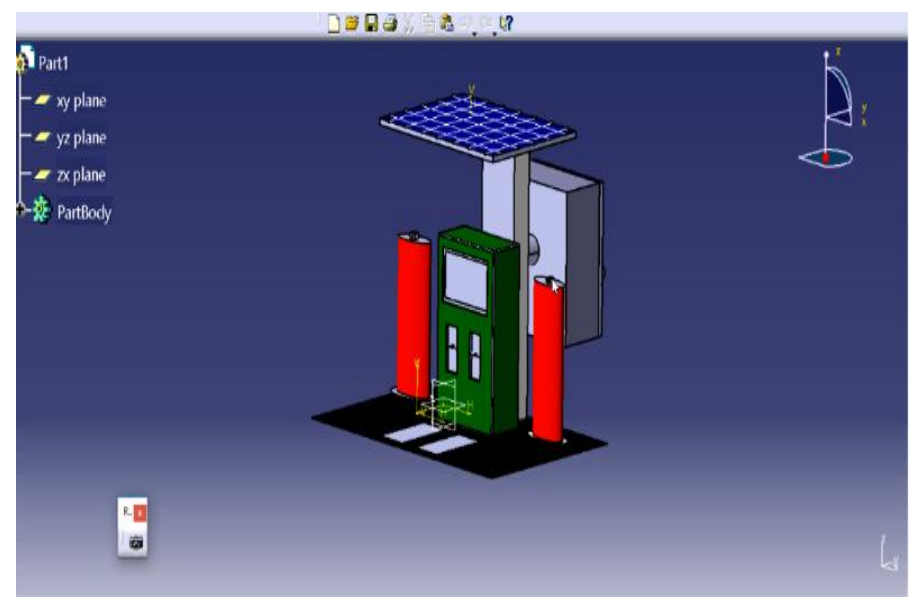

Fig. 1. Design of the entire Model

\section{MARKET RESEARCH}

According to our secondary market research we found that there are $279 \mathrm{AD}$ agencies in Dubai (U.A.E). Statistical data shows that there are almost 150,000 Businesses ranging from asmcs to mncs and every company has a fixed marketing budget. The DUBAI ELECTRICITY \& WATER AUTHORITY which is known as DEWA has around $200 \mathrm{EV}$ Chargers. So far, we have mutual agreements from $4 \mathrm{AD}$ 
agencies \& 1 company to use our services and they have subscribed to our website.

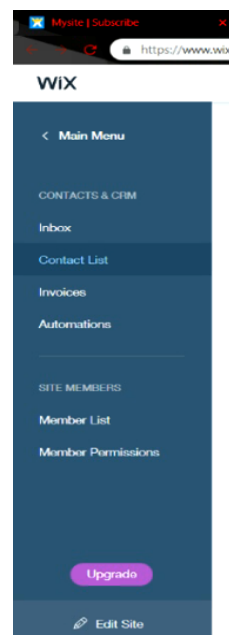

$\mathbf{x}$ as

Contact List: All Contacts

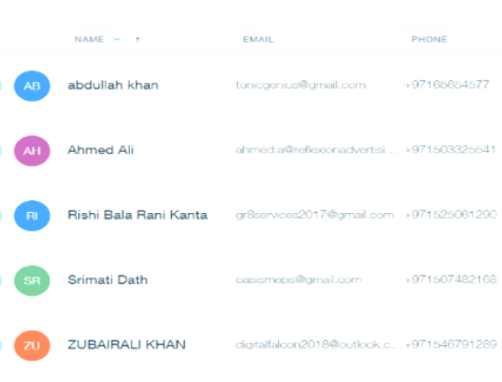

Fig. 2. List of contacts subscribed to our website

\section{RESULTS}

By March 2019 we had tie-ups with $4 \%$ of AD Agencies and by June 2019 it turned out to be $6 \%$

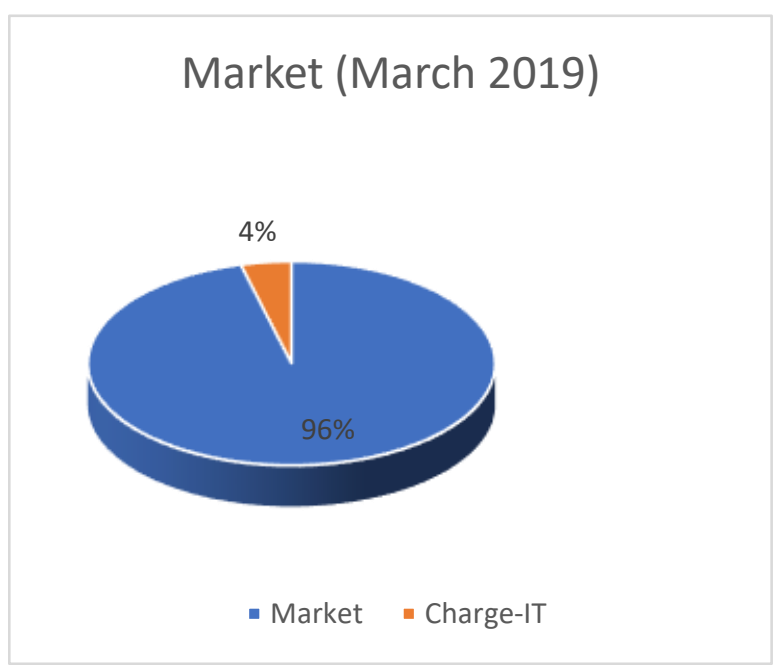

Fig. 3. Market of March 2019

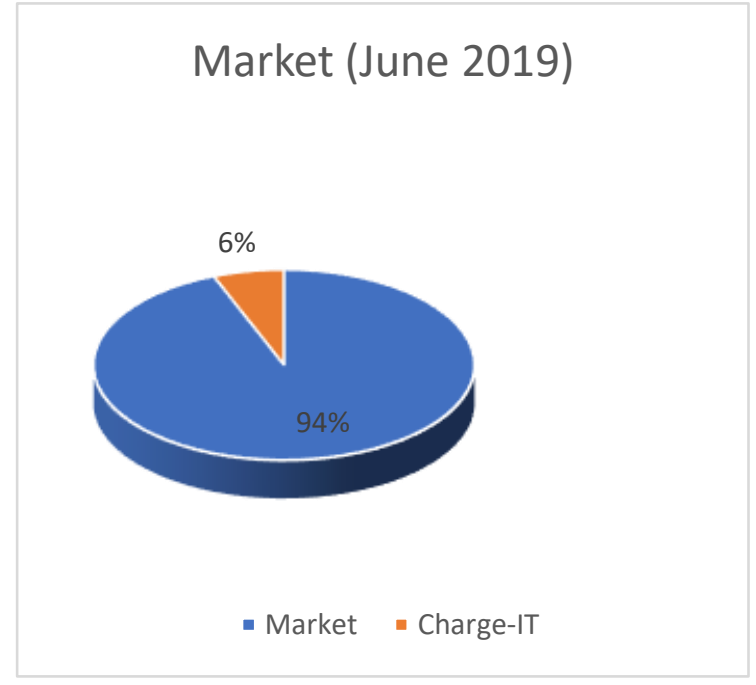

Fig. 4. Market of June 2019

\section{COSTS \& REVENUE MODEL}

We have the detailed lists of costs for manufacturing and installing our product.

1) Solar Panels $=750 * 4=$ AED 300

2) Inverter $=$ AED 750 a $2 \mathrm{Kw}$ system

3) LED Boards (2) - AED 20,000

4) Aluminum Structure - AED 2500

5) Cameras/ Sensors/Data Storage/WIFI Router - AED 5,000

Miscellaneous Costs (licensing, Construction, Installation etc.) - AED 10,000

Fixed - AED 25,000 would be born yearly which includes licensing and maintenance

Total - AED 66,250

2 Models:-

Starter Pack - Operates on monthly basis (1 month contract AED 8000) includes 2 hrs of advertisements per day on all AD Boards as well as 1 Newspaper AD.

Professional Pack - 3 Month contract (AED 23,000) and covers 3 hrs of Advertisements per day as well as 3 newspaper AD's 


\section{CONCLUSION}

This research work was set to develop our offering a novel solution to the growing market. By opening new paths of advertisements, we keep the audience hooked. With our new design and invention this has opened up new doors for ad agencies for investing into our product.

\section{REFERENCE}

[1] Data for market research was sourced from primary and secondary sources. (2018), SUSTAINABLE GREEN VENTURE.

[2] https://www.dsc.gov.ae/en-us (2018) Market Research Analysis.

[3] https://www.dewa.gov.ae/en/customer/innovation/smartinitatives/electrical-vehicle-charging-stations (2018) Market Research.

[4] Mentors whom we had contacted personally - Dr. Alfaz Khurram (2018) Advertisements Board with LED, , MD Mulk Holdings.

[5] Mr. Madi Aldabergen (2018) SUSTAINABLE GREEN VENTURE, Electric Vehicle Charger DUBAI ELECTRICITY \& WATER AUTHORITY.

[6] Mr. Nawab Afaque (2018) ELECTRIC VHICLE CHARGER DESIGN Solar engineer- Mulk Holdings

[7] Our Website Link SAI MAHAAN GANNAVARAPU \& PRANAY (2018) https://f20150270.wixsite.com/mysite

[8] Dr. Trupti Swarup Ghokale, (2014) "Stress-Tolerance of Sinorhizobium spp. Nodulating Sesbania and Cowpea in Desert Soils" Journal of Pure and Applied Microbiology, 8(1): 323-331, Associate Professor, Biotechnology, BITS Pilani, Dubai Campus, New Venture Creation Course Instructor.

[9] Dr. Mridula Goel, (2017), Enhancing the effectiveness of marketing a tourist destination using satisfaction analysis.(co authored with Pawaskar, P) 2017 International Journal of Business and Systems Research, 11(1/2), 163-181. Associate Professor, BITS Pilani, Goa Campus New Venture Creation, Course Instructor.

[10] Dr. Jyoti (2017) ICT enabled classroom effectiveness scale development and validation: A case of multicampus university", Knowledge Management \& ELearning, Vol.9, No.1, pp. 111-127, Asssociate Professor, BITS Pilani, Pilani Campus New Venture Creation.

[11] Dr. Vincent Shantha Kumar (2011), “Thermodynamic prediction of bulk metallic glass forming alloys in ternary $\mathrm{Zr}-\mathrm{Cu}-\mathrm{X}(\mathrm{X}=\mathrm{Ag}, \mathrm{Al}, \mathrm{Ti}, \mathrm{Ga})$ systems" (pp- 3495-3499) , Associate Professor, Mechanical Engineering, BITS Pilani, Dubai Campus.

[12] Dr. Rhagunathan (2018), Associate Professor, Economics $\&$ Finance, BITS Pilani, Hyderabad Campus.

\section{ACKNOWLEDGEMENTS}

We would like to thank our mentors, Dr. Alfaz Khurram, Mr. Nawab Afaque, Mr. Tai Al khairi who have expertise in advertisements and energy segments for making our product into reality. 